\title{
Phenomenon of relaxation of inhomogeneous elastic rotational curvature of the lattice of nanofine spatial dissipative structures of hexagonal selenium
}

\author{
V B Malkov ${ }^{1}$, O V Chemezov ${ }^{2,5}$, V G Pushin ${ }^{3}$, A V Malkov ${ }^{4}$, B V Shulgin ${ }^{2}$ and \\ O V Malkov ${ }^{4}$ \\ ${ }^{1}$ Institute of High-Temperature Electrochemistry, Ural Branch of the Russian \\ Academy of Sciences, 20, Academicheckaya str., Yekaterinburg, 620990, Russia \\ ${ }^{2}$ Ural Federal University named after the First President of Russia B N Yeltsin, 19, \\ Mira str., Yekaterinburg, 620000, Russia \\ ${ }^{3}$ Institute of Metal Physics named after M N Mikheeva Ural Branch of the Russian \\ Academy of Sciences, 18, Sophyi Kovalevskoy str., Yekaterinburg, 620108, Russia \\ ${ }^{4}$ CJSC SPC 'Rosna', Yekaterinburg, Russia \\ E-mail: ${ }^{5}$ o.v.chemezov@urfu.ru
}

\begin{abstract}
Nanofine rhomboid spatial dissipative structures (NRSDS) of hexagonal selenium were found and studied by means of transmission electron microscopy in amorphous Se films coated with nanofine layers of amorphous carbon, at a temperature of their thermo gradient treatment of $423 \mathrm{~K}$. As a result, pictures were received of fan-shaped linear bending extinction contours on the electron-microscopic images of NRSDS. It was shown that in the above NRSDS take place continuous linear increase in the bend radius of the lattice along [001], due to continuous linear relaxation of the inhomogeneous elastic torsion of the lattice around [001]. The continuous linear relaxation of the elastic rotational curvature of the lattice around [001] in NRSDS of hexagonal selenium with inhomogeneous elastic rotational curvature of the lattice covers the entire nanofine rhomboid spatial dissipative structures, and not its part, as is the case with the formation of interblock boundaries in the nanofine rhomboid spatial dissipative structures.
\end{abstract}

\section{Introduction}

It is known that the formation of interblock boundaries take place with varying module of the misorientation vector $\varpi$ in NRSDS of hexagonal selenium - nanofine crystals after the stage of quenching of NRSDS's [1-4]. This phenomenon is the result of relaxation of an inhomogeneous elastic rotational curvature of the lattice around [001] in one of NRSDS block [5-9]. At the same time, it is known that the formation of interblock boundaries in NRSDS, along which not only the modulus but also the sign of the misorientation vector changes, is a result of relaxation of the inhomogeneous elastic rotational curvature of the lattice around [001] in neighboring blocks of NRSDS [8,9]. The driving force of relaxation processes in NRSDS's of hexagonal selenium, leading to the formation of interblock boundaries with a crystal - geometric parameters varying along the boundaries, is the tendency to decrease the energy of the inhomogeneous elastic rotational curvature of their lattice around [001] [5-7]. The relaxation process of the inhomogeneous elastic rotational curvature of the lattice of NRSDS around [001], which determine the formation of the interblock torsion boundaries, 
are realized only in the part of NRSDS's of hexagonal selenium [5-9].

\section{Results and discussion}

The method of transmission electron microscopy and electron diffraction method [10-13] used to study the amorphous films of selenium, after thermo gradient processing by unilateral heating of their lower surface at a temperature of $423 \mathrm{~K}$. NRSDS of hexagonal selenium were discovered and investigated. Pictures of fan-shaped linear bending extinction contours are present on the electron microscopic images of the detected NRSDS. Let us consider the features of the mutual arrangement of bending extinction contours (Figure $1 \mathrm{a}$ ). It is noteworthy that the 'fan' of bending contours, the base of which is located in one of the blunt corners of the rhombus, is formed by rectilinear bending contours.

The bending contours deviate from the direction of the lowest formation speed towards the direction of the highest formation speed of NRSDS occur when moving take place along the direction of the lowest formation rate of NRSDS, which coincides with the short diagonal of a rhombus (Figure 1 a).

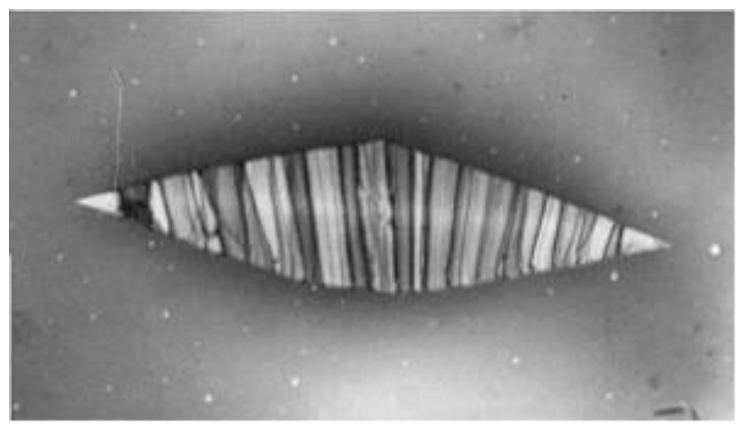

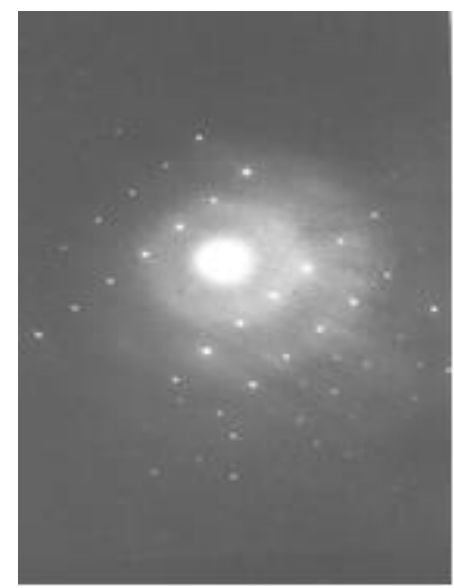

b

Figure 1. a) NRSDS of hexagonal selenium with a linear fan-shaped system of bending contours;

b) The microelectron diffraction pattern from the central part of NRSDS of hexagonal selenium. In a reflective position are prismatic planes of the first kind.

Bending contours deviating from the position parallel to [001] retain the shape of straight lines (Figure $1 \mathrm{a}$ ). The distance between the bending extinction contours, which differ only in the sign of the indices $h k l, \bar{h} \bar{k} l$ increase in the direction of the opening of the 'fan' of the contours. The distance between the centers of pairs of bending contours, the appearance of which on the electron microscopic images of NRSDS due to reflection from pairs of planes with different indices $h_{1} k_{1} l_{1}, \bar{h}_{1} \bar{k}_{1} \bar{l}_{1} ; h_{2} k_{2} l_{2}, \overline{h_{2}} \bar{k}_{2} \bar{l}_{2}$, increase in the same direction. The increase in the distances between bending contours that differ only in sign, and between the centers of pairs of bending contours with different indices, is also observed along the direction of the highest rate of formation of NRSDS. The similar change in the intercontour distances was previously observed for NRSDS of hexagonal selenium with inhomogeneous elastic rotational curvature of the lattice around [001] [14], which indicates the heterogeneity of the elastic rotational curvature of lattice in the studied NRSDS.

Electron diffraction patterns were obtained from the central part of the NRSDS of hexagonal selenium (Figure $1 \mathrm{~b}$ ) in electron diffraction studies. The ones coincide with electron diffraction patterns obtained from the central part of NRSDS of hexagonal selenium with systems of bending contours parallel to the short diagonal of the rhombus and inhomogeneous elastic curvature of the lattice around [001] [14]. The conclusion follows from here that the initial state for the formation of 
NRSDS with a linear fan-shaped system of bending extinction contours on electron microscopic images is the state of NRSDS of hexagonal selenium with inhomogeneous elastic rotational curvature of the lattice around [001] [14].

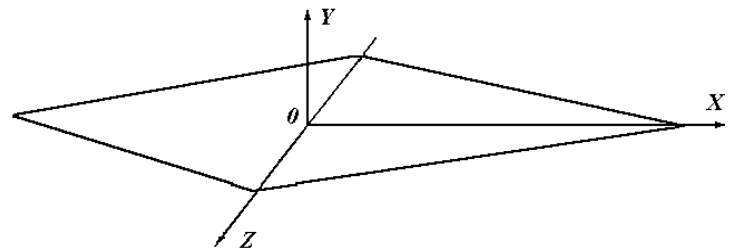

Figure 2. The coordinate system associated with NRSDS of hexagonal selenium.

It has been established that the lattice of NRSDS of hexagonal selenium (Figure 1 a) experiences an inhomogeneous elastic rotational curvature of the lattice around two mutually perpendicular directions - around [001], which coincides with the OZ axis (Figure 2) and around the direction of OX (Figure 2 ), which coincides with the long diagonal of the rhombus [15-18]. Calculations performed using standard crystallographic formulas show that the rotation of the lattice in the reference plane $(001)$ reaches $18^{\circ}$, and the deviation of [001] from a position parallel to the plane of the film reaches $22^{\circ}$. The features of the patterns of bending extinction contours on the electron microscopic images of NRSDS indicates that the rotational curvature of the lattice of NRSDS with a linear fan-shaped system of bending contours has continuous progressive nature. For a more complete study of the organization of the lattice of NRSDS (Figure 1 a), we used the formulas of the method of bending extinction contours $[19,20]$.

As it was established above, for NRSDS of hexagonal selenium with a linear fan-shaped system of bending contours in the electron microscope image, the increase in the inter - contour distances in the direction of the opening of the 'fan' of contours is observed both between pairs of contours that differ only in signs $h k l ; \bar{h} \bar{k} l$ and between the centers of pairs of contours with different indices $h_{1} k_{1} l_{1}, \overline{h_{1}} \bar{k}_{1} \bar{l}_{1} ; h_{2} k_{2} l_{2}, \overline{h_{2}} \overline{k_{2}} \bar{l}_{2}$. Let us consider how the lattice bending parameters of the above mentioned NRSDS are changed to realize the revealed change in the intercontour distances. For this purpose, we use the formulas for the distance between contours obtained in $[19,20]$. The formula for the distance between the pair of contours that differ only in signs $h k l ; \bar{h} \bar{k} \vec{l}$ :

$$
N^{(\beta)}=\frac{2 r \cdot \sin \varphi_{U V W}}{1+\sin ^{2} \beta \cdot \operatorname{ctg}^{2} \gamma}
$$

The formula for the distance between the centers of pairs of contours, the appearance of which on the electron microscopic image of the crystal is due to reflections from planes with different indices $h_{1} k_{1} l_{1}, \overline{h_{1}} \bar{k}_{1} \bar{l}_{1} ; h_{2} k_{2} l_{2}, \overline{h_{2}} \bar{k}_{2} \bar{l}_{2}$ :

$$
M^{(\beta)}=r \cdot \sqrt{1-\frac{1}{\sin ^{2} \beta \cdot \operatorname{ctg}^{2} \gamma}}
$$

Since, under the conditions of our experiment, the quantities $\sin \beta, \operatorname{ctg} \gamma, \sin \phi_{U V W}$ are constant, so far as formulas (1) and (2) can be transformed as follows:

$$
N^{(\beta)}=r \cdot c_{1}^{\prime} ; M^{(\beta)}=r \cdot c_{2}^{\prime}
$$

Having marked, $\frac{1}{c_{1}^{\prime}}=c_{1}, \frac{1}{c_{2}^{\prime}}=c_{2}$, we will receive: 


$$
r=c_{1} \cdot N^{(\beta)} ; r=c_{2} \cdot M^{(\beta)}
$$

From formula (3), it is seen that the dependence between the bending radius of the crystal lattice $r$ and distances, between contours that differ only in sign $h k l ; \bar{h} \bar{k} l$ and between the centers of the contour pairs, the appearance of which on the electron microscopic image of the crystal is due to reflections from planes with different indices $h_{1} k_{1} l_{1}, \bar{h}_{1} \bar{k}_{1} \bar{l}_{1} ; h_{2} k_{2} l_{2}, \overline{h_{2}} \bar{k}_{2} \bar{l}_{2}$ is linear. It is clear that this nature of the change in the intercontour distances is due to an increase in the bend radius of the lattice of NRSDS in the direction of the opening of the 'fan' of the contours. Given the straightforwardness and continuity of bending contours present on the electron microscopic images of NRSDS and formulas (3) [17], it is clear that the increase in the radius of the rotational curvature of the lattice will occur according to a linear law. The calculations performed using the formulas of the bending contour method $[19,20]$ and formula (3) confirm the above conclusion.

\section{Conclusion}

Thus, in the process of studying NRSDS of hexagonal selenium the features of their lattice organization were revealed. The bending radius of the lattice of these NRSDS increases in direction of contour 'fan' opening according to the linear law. The initial state for the formation of NRSDS with a linear fan-shaped system of bending contours is the state of the NRSDS of hexagonal selenium with inhomogeneous elastic rotational curvature of the lattice around [001]. The increase in the bending radius of the lattice of NRSDS in the direction of contours those are similar to an 'open fan' take place according to the linear law. This phenomenon can be explained as the result of continuous linear relaxation of the elastic rotational curvature of the lattice around [001] in the NRSDS of hexagonal selenium with inhomogeneous elastic rotational curvature of the lattice. In this case, the continuous linear relaxation of the elastic rotational curvature of the lattice around [001] in NRSDS of hexagonal selenium with inhomogeneous elastic rotational curvature of the lattice covers the entire NRSDS's, and not its part, as is the case with the formation of interblock boundaries in NRSDS's.

\section{Reference}

[1] Prigogine I and Condepudi D 2002 Modern thermodynamics From heat engines to dissipative structures (Moscow: Mir) [In Russian]

[2] Nikolis G and Prigozhin I 1979 Self-organization in nonequilibrium systems ed Chizmadzheva Yu A (Moscow: Mir) [In Russian]

[3] Malkov V B, Nikolaenko I V, Shveikin G P, Malkov A V, Pushin V G, Shulgin B V, Malkov O V and Plaksin S V 2017 Patent RU 2637396 [In Russian]

[4] Malkov V B, Nikolaenko I V, Shveikin G P, Pushin V G, Malkov A V, Malkov O V and Shulgin B V 2018 Formation of dissipative structures in an amorphous film Reports of the Academy of Sciences $\mathbf{4 7 8} 5$ pp 543-5

[5] Bolotov I E, Kolosov V Yu and Malkov V B 1984 Application of Bend-Contour Method the Investigation of Block Formation in Thin-Film Crystals Budapest Electron Microscopy 2 pp $42-5$

[6] Bolotov I E, Kolosov V Yu and Malkov V B 1986 Electron Microscope Investigation of Crystals Based on Bend-Contour Arrangement III Formation of Subgrain Boundaries in Dislocation-Free Crystals of selenium Phys. Stat. Sol. (a) 95 pp 377-83

[7] Bolotov I E, Kolosov V Yu and Malkov V B 1986 A new type of interblock boundaries in thinfilm selenium crystals Crystallography 311 pp 204-6 [In Russian]

[8] Malkov V B, Malkov A V, Pushin V G, Streckalovskiy V N and Malkov O V 2012 Patent RU 2534719 [In Russian]

[9] Malkov V B, Nikolaenko I V, Shveikin G P, Pushin V G, Malkov A V, Malkov O V and Shulgin B V 2018 The mechanism of the formation of interblock boundaries, the effect of changing the sign of the vector of orientation along dangling interblock boundaries in 
nanofine spatial dissipative structures Reports of the Academy of Sciences $\mathbf{4 8 1} 1$ pp 30-2

[10] Hirsch P B, Howie A, Nicholson R B and Pashley D W 1965 Electron Microscopy of Thin Crystals (London: Butterworths) pp 418-20

[11] Tomas G and Goringe M J 1979 Transmission electron microscopy of materials (New YorkChichester-Brisbane-Toronto) pp 198-203

[12] Utevsky L M 1973 Diffraction electron microscopy in metal science (Moscow: Metallurgy) [In Russian]

[13] Andrews K V, Dyson D J and Keown S R 1968 Interpretation of Electron Diffraction Patterns (London) pp 39-56

[14] Malkov V B, Malkov A V, Malkov O V, Pushin V G, Shulgin B V and Agalakov S P 2008 The phenomenon of elastic rotational curvature of the lattice of nanofine crystals of hexagonal selenium around [001] in the framework of the asymmetric theory of elasticity Collection of reports Kharkov Nanotechnological Assembly 2 (Kharkov Ukraine) pp18-23

[15] Malkov V B, Nikolaenko I V, Shveikin G P, Malkov A V, Pushin V G, Malkov O V and Shulgin B V 2014 Patent RU 2534719 [In Russian]

[16] Malkov V B, Nikolaenko I V, Shveikin G P, Malkov A V, Pushin V G, Shulgin B V and Malkov O V 2018 The lattice symmetry of nanofine crystals isomorphism of the forward and backward spaces and the Curie symmetry principle Reports of the Academy of Sciences $\mathbf{4 7 9}$ 1 pp $18-20$

[17] Malkov V B, Nikolaenko I V, Shveikin G P, Malkov A V, Pushin V G, Malkov O V and Shulgin B V 2015 Patent RU 2570106 [In Russian]

[18] Malkov V B, Nikolaenko I V, Shveikin G P, Malkov A V, Pushin V G, Shulgin B V, Malkov O V and Plaksin C V 2019 Patent RU 2687876

[19] Bolotov I E and Kolosov V Yu 1980 Bending of thin-film crystals of selenium detected by extinction contours Izv. USSR Academy of Sciences ser. physical 446 pp1194-97 [In Russian]

[20] Bolotov I E and Kolosov V Yu 1982 Investigation of Crystals Based on Bend-Contour Arrangement 1 Relationship between Bend-Contour Arrangement and Bend Geometry Phys. Stat. Sol. (a) 691 pp 85-96 